\title{
Diversidad florística de Araceae en el eStado de TABAsCo, MÉXICO
}

\author{
Pedro Díaz-Jiménez ${ }^{1}$, María de los Ángeles Guadarrama-Olivera ${ }^{2}$ y Thomas B. Croat ${ }^{3}$ \\ ${ }^{1}$ Centro de Investigaciones Tropicales, Universidad Veracruzana, Xalapa, Veracruz, México \\ ${ }^{2}$ Universidad Juárez Autónoma de Tabasco, División Académica de Ciencias Biológicas, \\ Villahermosa, Tabasco, México \\ ${ }^{3}$ Missouri Botanical Garden, St. Louis, Missouri, U.S.A. \\ ${ }^{1}$ Autor para la correspondencia: aroid764@hotmail.com
}

\begin{abstract}
Resumen: Se estudió la diversidad florística de la familia Araceae en el estado de Tabasco, México. Se encontraron un total de 44 taxa nativos (30 especies y 14 taxa infraespecíficos) en nueve géneros, que corresponden al 40\% de las 110 especies registradas para México. Nueve especies son nuevos registros para el estado y siete son endémicas de México. Los géneros con mayor número de especies fueron Philodendron (17 especies) y Anthurium (11), los cuales comprendieron el 63.6\% del total. El 75\% de las especies fueron hemi- y holoepífitas. Anthurium schlechtendalii subsp. schlechtendalii, Philodendron radiatum var. radiatum, Syngonium angustatum y $S$. podophyllum var. podophyllum se encontraron ampliamente distribuidas en los diversos tipos de vegetación de Tabasco. Anthurium pedatoradiatum subsp. helleborifolium se encontró en elevaciones de hasta $720 \mathrm{~m}$, elevación relativamente mayor en comparación con el intervalo altitudinal de esta especie reportada para el Neotrópico. Monstera tuberculata var. tuberculata es la única especie del estudio enlistada en la NOM-059-SEMARNAT-2010 como especie amenazada para México. El alto número de nuevos registros de Araceae reportado en este estudio, demuestra la importancia de la revisión de herbarios, misma que permitió corregir numerosas especies erróneamente identificadas y registradas en diferentes trabajos florísticos, así como la necesidad de intensificar exploraciones en campo, particularmente en los tipos de vegetación donde la familia se encontró mejor representada, tales como la selva alta perennifolia y selva mediana subperennifolia, en donde se registró el 59\% de las especies. Palabras clave: aráceas, clave de identificación, distribución geográfica, endemismo, riqueza de especies.
\end{abstract}

\begin{abstract}
We studied the floristic diversity of Araceae in the state of Tabasco, Mexico. We found 44 native taxa (30 species and 14 infraspecific taxa) in nine genera, corresponding to $40 \%$ of the 110 species recorded for Mexico. Nine species are recorded for the first time for Tabasco, and seven are endemic to Mexico. The largest genera were Philodendron (17 species), and Anthurium (11), which together accounted for $63.6 \%$ of the species. The hemi- and holoepiphytic aroids represented the $75 \%$ of all species. Anthurium schlechtendalii subsp. schlechtendalii, Philodendron radiatum var. radiatum, Syngonium angustatum and Syngonium podophyllum var. podophyllum were widely distributed in different vegetation types of Tabasco. Anthurium pedatoradiatum subsp. helleborifolium was found growing until $720 \mathrm{~m}$, which is a relatively higher elevation compared to the altitudinal range of this species reported for the Neotropics. Monstera tuberculata var. tuberculata is the only species in our study listed in NOM059-SEMARNAT-2010 as threatened in Mexico. The high number of new aroid records reported in this study demonstrates the importance of herbarium revisions, which allowed to correct many misidentified species recorded in different floristic studies, and the need of further intensive exploration in the field, particularly in vegetation types where the family is well represented, such as the tropical rain forest and semievergreen forest of Tabasco, where 59\% of the species were found.
\end{abstract}

Key words: aroids, endemism, geographical distribution, identification key, species richness.

a familia Araceae es una de las más diversas en la flora tropical, que en su sentido tradicional (excluyendo Acoraceae y Lemnaceae), está conformada por 105 géneros y aproximadamente 3,300 especies. Está distribuida principalmente en regiones tropicales y subtropicales, así como en las templadas del hemisferio norte (Croat y Carlsen, 2003). Se encuentran en una amplia variedad de hábitats, desde bosque seco tropical hasta bosque pluvial, pantanos subárticos, pantanos tropicales, bosques montañosos y planicies costeras áridas a semiáridas. La riqueza de especies es ma- 
yor en la zona tropical, entre el nivel del mar y elevaciones medias, hasta cerca de los 1,500 m (Croat, 1998). Dentro de la familia, las hemiepífitas; es decir, aquellas plantas que aunque crecen sobre un hospedero, tienen conexión con el suelo en al menos una etapa de su ciclo de vida (Nadkarni et al., 2001), son la forma de vida más diversa en comparación con las acuáticas, holoepífitas o epífitas verdaderas, rupícolas y terrestres (Croat, 1988). Para México se enlistan 110 especies en 13 géneros (Croat y Carlsen, 2003), que incluye una nueva especie de Monstera descrita recientemente (Croat et al., 2010); la mayoría de estas se distribuyen principalmente en la vertiente del Pacífico y del Golfo, con pocos hábitats disponibles para su crecimiento en la meseta central (Bunting, 1965). Los géneros con mayor número de especies en México son: Anthurium con un total de 41 especies, de las cuales 26 son endémicas y, Philodendron subg. Philodendron con 21 taxa, incluyendo siete endémicos (Croat, 1983, 1997, 1998).

Dentro de los estudios de exploración y colecta de los recursos vegetales en el estado de Tabasco, existen algunos trabajos que documentan la diversidad florística de las Araceae en zonas frecuentemente exploradas; por ejemplo, Bunting (1965) enlista 24 especies colectadas en tres municipios (Teapa, Tacotalpa y Tenosique); Ramón-Jiménez (1992), al estudiar la flora de angiospermas epífitas en la vegetación riparia del río Puyacatengo (Teapa), reporta un total de 12 especies de Araceae epífitas; López-Hernández (1994), en su estudio de la vegetación y la flora de la Sierra de Tabasco, en los municipios de Tacotalpa y Teapa, enlista 31 especies distribuidas en ocho géneros; Hanan-Alipi (1997) reporta un total de 24 especies (15 hemi- y holoepífitas, nueve rupícolas y/o terrestres) localizadas en la Sierra del Madrigal del municipio de Teapa; y Díaz-Jiménez (2006b), en su estudio sobre la composición florística de la familia Araceae en el desarrollo Ecoturístico Kolem jaa' del municipio de Tacotalpa, reporta 20 especies. Existen otros estudios más generales donde se mencionan un mayor número de especies para todo el estado de Tabasco: Cowan (1983) enlista 40 especies y en Pérez et al. (2005) se reconocen 44 especies, de las cuales sólo 27 y 26, respectivamente, fueron corroboradas en este estudio y las demás eran sinónimos y material erróneamente identificado.

La amenaza principal para la supervivencia a largo plazo de la familia Araceae es la pérdida y la reducción de sus hábitats naturales (Mayo et al., 1997). En el estado de Tabasco, la mayoría de los ecosistemas naturales han sido modificados y continúan reduciéndose debido a diversas actividades antropogénicas, como la deforestación, la expansión ganadera extensiva, la intensificación agrícola, la urbanización y la explotación petrolera (Pérez et al., 2005). El $90 \%$ de la vegetación arbórea original en el estado ha sido deforestada, permaneciendo un resto generalmente en lugares inundables y/o inaccesibles por su topografía accidentada (López, 1980); sin embargo, las Araceae continúan siendo abundantes en algunos fragmentos de selvas que presentan poca alteración. El presente trabajo tuvo como finalidad contribuir con una amplia revisión de ejemplares de herbario y un estudio florístico para presentar un listado actualizado, incluyendo una clave dicotómica de identificación de la familia Araceae (Apéndice 1) en el estado de Tabasco, al analizar algunos aspectos sobre su distribución y ecología, tales como: formas de vida, distribución altitudinal, distribución en el Neotrópico y en los diferentes tipos de vegetación presentes en el estado.

\section{Materiales y métodos}

Área de estudio. El estado de Tabasco se localiza al SE de México, colinda al norte con el Golfo de México, al este con Campeche y la República de Guatemala, al sur con Chiapas y al oeste con el estado de Veracruz (Figura 1). Está situado entre las coordenadas $17^{\circ} 19^{\prime}$ y $18^{\circ} 39^{\prime}$ de latitud norte y $90^{\circ} 57^{\prime}$ y $94^{\circ} 08^{\prime}$ de longitud oeste. El relieve es casi plano en la mayoría de su territorio, con numerosas depresiones que forman cuerpos de agua y, hacia el sur, una porción de pequeñas sierras en los municipios de Huimanguillo, Macuspana, Tacotalpa, Teapa y Tenosique, cuyas altitudes se encuentran entre los 40 y 900(-1,000) m. El clima es cálido húmedo con abundantes lluvias en verano (junio-octubre) en la mayor parte de su territorio, comúnmente provocada por los ciclones tropicales del Golfo de México (Salazar, 1994), una temperatura media anual de $26^{\circ} \mathrm{C}$ y una precipitación anual entre $1,500 \mathrm{~mm}$ en la costa y $4,000 \mathrm{~mm}$ hacia la sierra (INEGI, 2012). En el estado se encuentran diversos tipos de vegetación de acuerdo a la clasificación de López (1980), tales como: selva alta perennifolia de ramón (Brosimum alicastrum); selva alta perennifolia de canshán (Terminalia amazonia) y chakté (Sweetia panamensis); selva alta perennifolia de canshán, ramón y huapaque (Dialium guianense); selva alta y mediana subperennifolia de ramón; selva mediana subperennifolia de ramón y huapaque, selva mediana subperennifolia de pukté (Bucida buceras); selva mediana

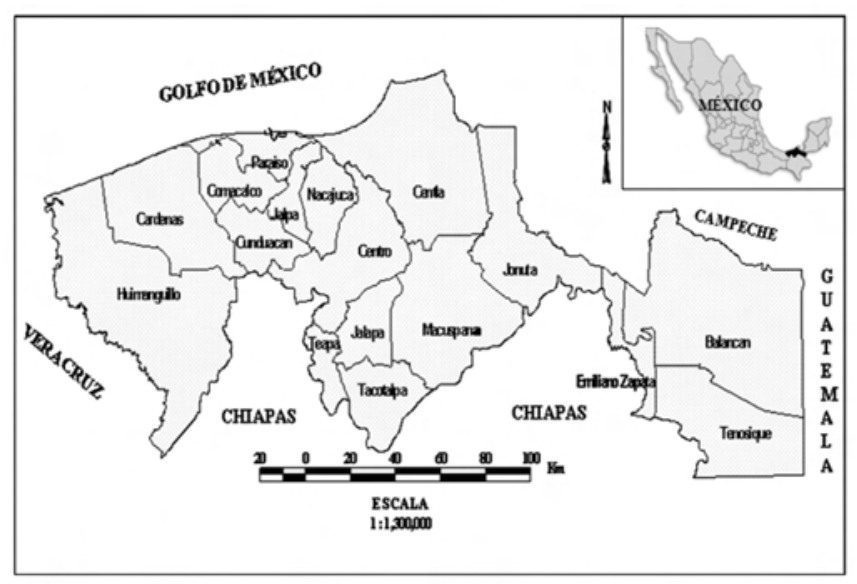

Figura 1. Mapa del estado de Tabasco y sus municipios, México. 
subperennifolia de chicozapote (Manilkara zapota) y pukté; selva mediana perennifolia de canacoite (Bravaisia integerrima); selva mediana subperennifolia de corozo (Attalea liebmannii); selva mediana subperennifolia de corozo, guano yucateco (Sabal yucatanica) y huapaque; selva baja espinosa perennifolia de tinto (Haematoxylum campechianum); encinar tropical (Quercus oleoides); sabana de jícaro (Crescentia cujete); sabana de tachicón (Curatella americana) y nance (Byrsonima crassifolia); comunidades de hidrófitas, manglar y vegetación riparia.

Trabajo de campo y de herbario. Para la realización del presente estudio se hizo una revisión de 788 ejemplares de la familia Araceae en los herbarios CSAT (Colegio de Postgraduados Campus Tabasco), MEXU (Universidad Nacional Autónoma de México), UJAT (Universidad Juárez Autónoma de Tabasco) y XAL (Instituto de Ecología, A.C.). Del 2003 al 2012 se hicieron 632 colectas de muestras botánicas usando el método descrito por Lot y Chiang (1986), en los diversos tipos de vegetación, abarcando los 17 municipios del estado. Se consideraron todas las formas de vidas presentes en la familia: acuáticas flotantes, hemiepífitas, holoepífitas, rupícolas y terrestres. El material colectado se herborizó, etiquetó, y con el uso de claves de identificación taxonómica y monografías del grupo (Bunting, 1960, 1965; Madison, 1977; Croat, 1978, 1981, 1983, 1997, 2004; Grayum, 1996; Mayo et al., 1997), se procedió a su determinación, posteriormente se depositó en el Herbario UJAT y se enviaron duplicados a los herbarios CSAT, MEXU y XAL, así como al Missouri Botanical Garden (MO), E.U.A. Cabe mencionar que, la identificación del mayor número de ejemplares colectados durante el presente estudio fue corroborada por el especialista en la familia Araceae, Thomas B. Croat, que es uno de los autores de este trabajo.

\section{Resultados}

La lista de Araceae para el estado de Tabasco incluye 44 taxa nativos (30 especies y 14 taxa infraespecíficos), en nue- ve géneros (Apéndice 2), de los cuales siete especies son endémicas de México y nueve son nuevos registros para el estado. Los géneros con mayor número de especies son Philodendron (17) y Anthurium (11). En cuanto a la forma de vida, 28 especies son hemiepífitas, siete terrestres, cinco holoepífitas, dos rupícolas-terrestres y una sola especie es acuática flotante y rupícola, respectivamente.

En relación con el tipo de vegetación, 37 especies fueron registradas para la selva alta perennifolia, diez de las cuales fueron exclusivas de este tipo de vegetación, y 30 especies para la selva mediana subperennifolia. En total 26, especies fueron compartidas entre ambos ambientes (Apéndice 2). Las especies con distribución más amplia en los diversos tipos de vegetación del estado de Tabasco fueron: Anthurium schlechtendalii subsp. schlechtendalii, Syngonium angustatum, Philodendron radiatum var. radiatum y $S$. podophyllum var. podophyllum, las dos primeras presentes en ocho de los nueve tipos de vegetación y las dos últimas en siete ambientes.

En el presente estudio, Anthurium pedatoradiatum subsp. helleborifolium se encontró en elevaciones mayores de hasta $720 \mathrm{~m}$. En relación con la distribución geográfica, solo la especie Pistia strationes es pantropical, 18 especies se distribuyen en México y Centroamérica, 14 hasta Sudamérica y siete son endémicas de México: A. faustomirandae, A. pedatoradiatum subsp. helleborifolium, Monstera egregia, Philodendron escuintlense, $P$. hederaceum var. oxycardium, $P$. seguine subsp. lingua-bovis y Syngonium neglectum.

\section{Discusión}

De acuerdo al presente estudio, el estado de Tabasco contiene el $40 \%$ de las 110 especies de la familia enlistadas para México (Croat y Carlsen, 2003; Croat et al., 2010). El número de especies es menor comparado con otras localidades neotropicales que se presentan a menor latitud, superficie e intervalo altitudinal, pero con una mayor precipitación, como es el caso de Cabo Corrientes, departamento del Chocó, Colombia (Cuadro 1). En general, la diversidad

Cuadro 1. Número de géneros y especies de Araceae de algunos territorios neotropicales, indicando su elevación, precipitación anual y superficie.

\begin{tabular}{|c|c|c|c|c|c|}
\hline & $\begin{array}{l}\text { Isla de Barro } \\
\text { Colorado, Panamá }\end{array}$ & $\begin{array}{l}\text { Estación Biológica } \\
\text { La Selva, Costa Rica }\end{array}$ & $\begin{array}{l}\text { Cabo Corrientes, } \\
\text { Chocó, Colombia }\end{array}$ & $\begin{array}{c}\text { Reserva de la } \\
\text { Biosfera Los Tuxtlas, } \\
\text { Veracruz, México }\end{array}$ & $\begin{array}{c}\text { Estado de Tabasco, } \\
\text { México }\end{array}$ \\
\hline Intervalo latitudinal & $9^{\circ} 8^{\prime}-9^{\circ} 11^{\prime}$ & $10^{\circ} 24^{\prime}-10^{\circ} 26^{\prime}$ & $5^{\circ} 28^{\prime}-5^{\circ} 34^{\prime}$ & $18^{\circ} 05^{\prime}-18^{\circ} 43^{\prime}$ & $17^{\circ} 19^{\prime}-18^{\circ} 39^{\prime}$ \\
\hline Elevación (m s.n.m.) & $0-145$ & 20-129 & $0-120$ & $0-1,700$ & $0-900(-1,000)$ \\
\hline Precipitación (mm) & 2,750 & 4,000 & 5,000 & 4,000 & $1,500-4,000$ \\
\hline Superficie $\left(\mathrm{km}^{2}\right)$ & 15.6 & 16 & 90 & 6.44 & 24,731 \\
\hline Número de géneros & 14 & 14 & 14 & 9 & 9 \\
\hline Número de especies & 51 & 97 & 114 & 34 & 44 \\
\hline Referencia: & Croat (1978) & McDade et al. (1994) & Mora et al. (2006) & Acebey y Krömer (2008) & Presente estudio \\
\hline
\end{tabular}


de Araceae es mayor en tierras bajas y húmedas cercanas al ecuador, la cual está relacionada con condiciones climáticas de alta precipitación y temperatura, así como a la ausencia de estaciones secas prolongadas (Mayo et al., 1997; Croat, 1998). Únicamente en la Reserva de la Biosfera "Los Tuxtlas", ubicada en el estado vecino de Veracruz, hay menos especies que en Tabasco, aunque en una superficie mucho menor (Acebey y Krömer, 2008). Ambos territorios comparten 27 especies, lo que demuestra una alta similitud florística.

Los géneros Anthurium y Philodendron comprendieron el $63.6 \%$ del total de las especies. Ambos géneros son los más grandes dentro de las Araceae a nivel mundial, con más de 1,000 y 700 especies, respectivamente (Croat, 1986, 1997). Las hemi- y holoepífitas fueron las formas de vida más numerosas, con el $75 \%$ del total de especies reportadas. Ambas formas de vida son las más diversas de la familia Araceae, en comparación con las terrestres; Philodendron es en gran parte hemiepífito con unas pocas especies terrestres y Anthurium comprende en su mayoría epífitas verdaderas (Croat, 1988, 1997).

El mayor número de especies (59\%) se encontró en selva alta perennifolia y selva mediana subperennifolia. Croat (1998) menciona que las Araceae hemi- y holoepífitas comprenden el mayor número especies en el Neotrópico y su mayor diversidad ocurre principalmente en los bosques o selvas húmedas. Las especies Anthurium schlechtendalii subsp. schlechtendalii, Philodendron radiatum var. radiatum, Syngonium angustatum y S. podophyllum var. podophyllum, se encontraron en diversos tipos de vegetación. Philodendron radiatum var. radiatum y $S$. podophyllum var. podophyllum presentan una amplia distribución, desde México hasta Sudamérica (Croat, 1981, 1997), mientras que A. schlechtendalii subsp. schlechtendalii pertenece a la sección Pachyneurium, la cual agrupa especies que toleran

Cuadro 2. Lista de especies erróneamente identificadas, sinónimos enlistados, referencia y/o espécimen examinado, y especie revisada y corregida en el presente trabajo.

\begin{tabular}{|c|c|c|}
\hline Especie erróneamente identificada & Referencia y/o espécimen examinado & Especie revisada y corregida en este trabajo \\
\hline Anthurium lezamae Matuda & $\begin{array}{l}\text { C. Cowan, M.A. Magaña y S. Zamudio } \\
3402 \text { (CSAT) }\end{array}$ & Anthurium berriozabalense Matuda \\
\hline $\begin{array}{l}\text { Anthurium podophyllum (Schltdl. } \\
\text { \& Cham.) Kunth }\end{array}$ & C. Cowan y S. Zamudio 3364 (CSAT) & $\begin{array}{l}\text { Anthurium pedatoradiatum subsp. } \\
\text { helleborifolium (Schott) Croat }\end{array}$ \\
\hline $\begin{array}{l}\text { Anthurium bakeri Hook. f. } \\
\text { Anthurium crassinervium (Jacq.) Schott } \\
\text { Anthurium joseanum Engl., sinónimo } \\
\text { de A. protensum Schott }\end{array}$ & $\begin{array}{l}\text { E. Moreno y O. Castillo } 069 \text { (CSAT); } \\
\text { Jim Conrad, R. Conrad y D. Rodríguez } \\
2866 \text { (CSAT); M.A. Magaña y S. } \\
\text { Zamudio } 237 \text { (CSAT); P. Valdivia } 2028 \text { (XAL) }\end{array}$ & $\begin{array}{l}\text { Anthurium schlechtendalii Kunth } \\
\text { subsp. schlechtendalii }\end{array}$ \\
\hline Dieffenbachia seguine (Jacq.) Schott & C. Cowan 2063 (CSAT) & Dieffenbachia oerstedii Schott \\
\hline $\begin{array}{l}\text { Monstera deliciosa Liebm. } \\
\text { Monstera pertusa (L.) de Vriese, sinónimo } \\
\text { de Monstera adansonii Schott }\end{array}$ & $\begin{array}{l}\text { G. Ruíz } 40 \text { (CSAT); H. Puig } 348 \\
\text { (CSAT); J.R. Díaz } 7 \text { (CSAT) }\end{array}$ & Monstera acuminata K. Koch \\
\hline Philodendron scandens C. Koch et Sello & C. Cowan 1999 (CSAT) & Philodendron jacquinii Schott \\
\hline $\begin{array}{l}\text { Philodendron latisagittium Matuda, } \\
\text { sinónimo de P. mexicanum Engl. }\end{array}$ & H. Puig 838 (CSAT) & Philodendron smithii Engl. \\
\hline $\begin{array}{l}\text { Spathiphyllum phryniifolium Schott } \\
\text { Spathiphyllum blandum Schott }\end{array}$ & $\begin{array}{l}\text { G. Ramos } 699 \text { (UJAT); C. Cowan } 2989 \\
\text { (CSAT); M.A. Magaña y S. Zamudio } \\
140 \text { (CSAT) }\end{array}$ & $\begin{array}{l}\text { Spathiphyllum cochlearispathum } \\
\text { (Liebm.) Engl. }\end{array}$ \\
\hline $\begin{array}{l}\text { Syngonium donnell-smithii Engl., } \\
\text { sinónimo de S. salvadorense Schott }\end{array}$ & J. Cantú 176 (CSAT) & $\begin{array}{l}\text { Syngonium podophyllum Schott } \\
\text { var. podophyllum }\end{array}$ \\
\hline $\begin{array}{l}\text { Sinónimos enlistados } \\
\text { Anthurium aemulum Schott }\end{array}$ & Pérez et al. (2005); Cowan (1983) & $\begin{array}{l}\text { A. pentaphyllum var. bombacifolium } \\
\text { (Schott) Madison }\end{array}$ \\
\hline Philodendron apocarpum Matuda & Pérez et al. (2005); Cowan (1983) & Philodendron jacquinii Schott \\
\hline Philodendron fenzlii Engl. & Pérez et al. (2005) & Philodendron tripartitum (Jacq.) Schott \\
\hline Philodendron oxycardium Schott & Pérez et al. (2005); Cowan (1983) & $\begin{array}{l}\text { Philodendron hederaceum var. } \\
\text { oxycardium (Schott) Croat }\end{array}$ \\
\hline Philodendron scandens K. Koch \& Sello & Pérez et al. (2005); Cowan (1983) & $\begin{array}{l}\text { Philodendron hederaceum (Jacq.) } \\
\text { Schott var. hederaceum }\end{array}$ \\
\hline Spathiphyllum lacustre Lundell & Pérez et al. (2005); Cowan (1983) & $\begin{array}{l}\text { Spathiphyllum cochlearispathum } \\
\text { (Liebm.) Engl. }\end{array}$ \\
\hline
\end{tabular}


áreas con una pronunciada estación seca (Croat, 1986), se distribuye en México y Centroamérica, al igual que $S$. angustatum. Ambas especies crecen en diversos ambientes, desde el nivel del mar hasta los 1,600 y $700(-1,200) \mathrm{m}$, respectivamente (Croat, 1981, 1983).

Con el presente estudio se amplió el intervalo altitudinal de Anthurium pedatoradiatum subsp. helleborifolium para el Neotrópico. Anthurium pedatoradiatum subsp. helleborifolium, especie endémica de Chiapas y Tabasco, fue colectada en elevaciones mayores (720 m), antes solo había sido reportada en elevaciones por debajo de los $550 \mathrm{~m}$ de altitud (Croat, 1983). Esta especie pertenece a la sección Schizoplacium, la cual contiene únicamente seis taxa descritos, tres de los cuales son endémicos de México (Croat, 1986). Sin embargo, nuevos datos moleculares indican que $A$. pedatoradiatum, así como otras especies, deben ser excluidas de la sección Schizoplacium y ser incluidas en la sección Dactylophyllium (Croat y Carlsen, 2013). Además, el presente estudio permitió corregir numerosas especies erróneamente identificadas y enlistadas en diversos trabajos florísticos, así como sinónimos de muchas especies (Cuadro 2).

Las siguientes especies son nuevos registros para la flora de Tabasco: Anthurium berriozabalense y A. faustomirandae habían sido colectadas solo en el estado de Chiapas (Croat, 1983; Pérez-Farrera y Croat, 2001); A. huixtlense, A. lucens, Philodendron seguine subsp. lingua-bovis y $P$. standleyi solo habían sido reportadas para los estados de Chiapas, Oaxaca y Veracruz (Croat, 1983; Grayum, 1996; Acebey y Krömer, 2008); Monstera egregia había sido citada por Madison (1977) como sinónimo de M. lechleriana, actualmente los especialistas en la familia han considerado retomar el nombre de $M$. egregia para las poblaciones mexicanas (Acebey y Krömer, 2008), esta especie anteriormente era conocida solo para los estados de Oaxaca y Veracruz (Madison, 1977); P. escuintlense reportada para Chiapas y Veracruz (Grayum, 1996) y por último, P. glanduliferum subsp. glanduliferum conocida solamente para Oaxaca (Croat, 1997).

\section{Conclusiones}

El alto número de nuevos registros de Araceae para el estado de Tabasco reportados en el presente estudio, demuestra la importancia de la revisión de herbarios, misma que permitió corregir numerosas especies erróneamente identificadas y registradas en diferentes trabajos florísticos, así como la necesidad de intensificar muestreos, principalmente en fragmentos de los tipos de vegetación donde la familia se encuentra ampliamente representada, así como en áreas poco exploradas, como en los municipios de Huimanguillo y Tenosique, en donde se colectó el mayor número de nuevos registros para el estado, y más aun considerando el acelerado cambio que sufren estos ecosistemas, principalmente por las actividades humanas.
Por otro lado, Anthurium pedatoradiatum subsp. helleborifolium es una especie endémica de Tabasco y Chiapas, y Philodendron radiatum var. radiatum representa una especie de importancia comercial y cultural para algunas comunidades étnicas del estado (Díaz-Jiménez, 2006a). Actualmente, Monstera tuberculata var. tuberculata es la única especie que se encuentra enlistada en la NOM-059-SEMARNAT2010 como amenazada para México, a pesar de que varias especies parecen ser raras y potencialmente en peligro en Tabasco, lo cual incrementa aun más la importancia de sumar esfuerzos para el desarrollo de estrategias enfocadas hacia la conservación de la biodiversidad en todo el estado.

\section{Agradecimientos}

Al personal de los herbarios CSAT, MEXU, UJAT y XAL, por las facilidades otorgadas para la revisión del material, en especial a Nahúm Muñiz Chavarría (UJAT) y Eustolia García- López (CSAT). A Wilfrido Miguel Contreras Sánchez, quien apoyó la presentación de avances del presente estudio en diferentes congresos nacionales e internacionales, así como a Trinidad Magaña- Ramírez, Andrés Manuel de la Cruz- López, Mario Eduardo Sosa y Edgar Alejandro Garduza- Rodríguez, por su gran apoyo en las salidas de campo. A Michael H. Grayum y Amparo Acebey por su gran ayuda para la elaboración y revisión de las claves de identificación de géneros y especies. A Thorsten Krömer, Heiko Hentrich, Leyda Rodríguez y a dos revisores anónimos por sus valiosos comentarios y sugerencias para mejorar el presente manuscrito.

\section{Literatura citada}

Acebey A. y Krömer T. 2008. Diversidad y distribución de Araceae de la Reserva de la Biosfera Los Tuxtlas, Veracruz, México. Revista Mexicana de Biodiversidad 79:465-471.

Bunting G.S. 1960. A revision of Spathiphyllum (Araceae). Memoirs of the New York Botanical Garden 10:1-54.

Bunting G.S. 1965. Commentary on Mexican Araceae. Gentes Herbarum 9:291-382.

Cowan C.P. 1983. Listados florísticos de México. I. Flora de Tabasco. Instituto de Biología, Universidad Nacional Autónoma de México, México, D.F.

Croat T.B. 1978. Flora of Barro Colorado Island. Stanford University Press, Palo Alto.

Croat T.B. 1981. A revision of Syngonium (Araceae). Annals of the Missouri Botanical Garden 68:565-651.

Croat T.B. 1983. A revision of the genus Anthurium (Araceae) of Mexico and Central America. Part I: Mexico and Middle America. Annals of the Missouri Botanical Garden 70:211-420.

Croat T.B. 1986. The distribution of Anthurium (Araceae) in Mexico Middle America and Panama. Selbyana 9:94-99.

Croat T.B. 1988. Ecology and life forms of Araceae. Aroideana 11:4-55.

Croat T.B. 1997. A revision of Philodendron subgenus Philodendron (Araceae) for Mexico and Central America. Annals of the Missouri Botanical Garden 84:311-704. 
Croat T.B. 1998. Tropical aroids: taxonomy, diversity and ecology. En: Mathew P. y Sivadasan M. Eds. Diversity and Taxonomy of Tropical Flowering Plants, pp. 235-286, Mentor, Calicut.

Croat T.B. 2004. Revision of Dieffenbachia (Araceae) of Mexico, Central America and the West Indies. Annals of the Missouri Botanical Garden 91:668-772.

Croat T.B. y Carlsen M. 2003. Araceae. Flora del Bajío y de Regiones Adyacentes. 114:1-37.

Croat T.B. y Carlsen M. 2013. A reassessment of Anthurium species with palmately divided leaves, and a reinterpretation of Anthurium section Dactylophyllium (Araceae). PhytoKeys 23: 41-54.

Croat T.B., Krömer T. y Acebey A. 2010. Monstera florescanoana (Araceae), a new species from central Veracruz, Mexico. Revista Mexicana de Biodiversidad 81:225-228.

Díaz-Jiménez P. 2006a. Motusay-Philodendron radiatum Schott (Araceae). Aroideana 29:158-160.

Díaz-Jiménez P. 2006b. Composición florística de la familia Araceae en el desarrollo Ecoturístico Kolem jaa' en Tacotalpa, Tabasco, México. Kuxulkab’ 12:17-20.

Grayum M.H. 1996. Revision of Philodendron subgenus Pteromischum (Araceae) for Pacific and Caribbean Tropical America. Systematic Botany Monographs 47:1-233.

Hanan-Alipi A.M. 1997. Análisis florístico de la Sierra El Madrigal, Teapa, Tab. Tesis de licenciatura, Facultad de Ciencias, Universidad Nacional Autónoma de México, México, D.F. 50 pp.

INEGI. Instituto Nacional de Estadística y Geografía. 2012. Perspectiva Estadística: Tabasco. Instituto Nacional de Estadística y Geografía, Aguascalientes.

Lot A. y Chiang F. 1986. Manual de Herbario. Consejo Nacional de la Flora de México, A. C., México, D.F.

López-Hernández E.S. 1994. La vegetación y la flora de la Sierra de Tabasco (Municipios de Tacotalpa y Teapa) México. Universidad Juárez Autónoma de Tabasco, División Académica de Ciencias Biológicas, Centro de Investigación para la Conservación de Especies Amenazadas, Departamento de Ecología y Conservación de Ecosistemas Tropicales, Villahermosa.
López M.R. 1980. Tipos de vegetación y su distribución en el estado de Tabasco y norte de Chiapas. Colección Cuadernos Universitarios Serie Agronómica No. 1. Universidad Autónoma Chapingo, Centro Regional Tropical Puyacatengo, Dirección de Difusión Cultural, Puyacatengo.

Madison M. 1977. A revision of Monstera (Araceae). Contributions from the Gray Herbarium 207:3-100.

Mayo S.J., Bogner J. y Boyce P.C. 1997. The genera of Araceae. Royal Botanic Gardens, Kew, Londres.

McDade L.A., Bawa K.S., Hespenheide H.A. y Hartshorn G.S. 1994. La Selva: Ecology and Natural History of a Neotropical Rain Forest. University of Chicago Press, Chicago.

Mora M., Bernal R., Croat T. y Jácome J. 2006. A phytogeographic analysis of Araceae of Cabo Corrientes (Chocó, Colombia) and comparable lowland tropical American floras. Annals of the Missouri Botanical Garden 93:359-366.

Nadkarni, N.M., Merwin M.C. y Nieder J. 2001. Forest canopies, plant diversity. En: Encyclopedia of Biodiversity, Vol. 3, pp. 27 40, Academic Press, San Diego.

Pérez-Farrera M.A. y Croat T.B. 2001. A new species of Anthurium (Araceae) from Chiapas, Mexico. Novon 11:88-91.

Pérez L.A., Sousa S.M., Hanan A.M., Chiang F. y Tenorio P. 2005. Vegetación terrestre. En: Bueno J., Álvarez F. y Santiago S. Eds. Biodiversidad del Estado de Tabasco, pp. 65-110, Instituto de Biología, Universidad Nacional Autónoma de México, Comisión Nacional para el Conocimiento y Uso de la Biodiversidad, México, D.F.

Ramón-Jiménez.V. 1992. Flora de angiospermas epífitas en la vegetación del rio Puyacatengo. Teapa Tabasco, México. Tesis de Licenciatura, Universidad Juárez Autónoma de Tabasco, Tabasco. 52 pp.

Salazar T.J.C. 1994. Compendio monográfico de la historia de Tabasco: Obra literaria pedagógica. $2^{\mathrm{a}}$ ed. Editado por el autor, Villahermosa.

Tropicos. 2012. Missouri Botanical Garden. <www.tropicos.org> (consultado 4 de enero 2013).

Recibido: 15 de diciembre de 2013

Aceptado: 22 de marzo de 2014 
Apéndice 1. Clave para la identificación de géneros y especies de Araceae de Tabasco

1. Plantas acuáticas flotantes con hojas esponjosas

Pistia stratiotes

1. Plantas terrestres, epífitas o hemiepífitas, nunca acuáticas flotantes.

2. Espádice uniforme, flores bisexuales, espata libre (sin envolver totalmente al espádice solo en los brotes).

3. Plantas terrestres, nunca epífitas o hemiepífitas; láminas oblongas, mucho más largas que anchas, venación paralelapinnadas; pecíolos conspicuamente envainados; espata generalmente ovada, a veces elíptica, espádice con 4-5 segmentos del perianto Spathiphyllum

4. Base de la lámina foliar anchamente obtusa a subcordada; pecíolos y vaina densamente punteados, vaina ondulada; pistilos apróx. dos veces más largos que el perianto, muy protuberantes alargados ............................S. coclearispathum 4. Base de la lámina foliar aguda o aguda-acuminada, pecíolos no punteados, con la vaina no undulada; pistilos igualmente tan largos que el perianto o más largos. S. blandum

3. Plantas terrestres, epífitas o hemiepífitas.

5. Pecíolos envainados solo cerca de la base; usualmente láminas cordiformes, ligeramente más largas que anchas; venación prominentemente reticulada; raíces emergiendo de entre los pecíolos; espata generalmente lanceolada, a veces ovada; espádice con 4 tépalos Anthurium

6. Láminas foliares prominentemente palmatífidas a palmatisectas.

7. Láminas foliares con todos los segmentos libres hasta la base. .A. pentaphyllum var. bombacifolium

7. Láminas foliares con los segmentos unidos en la base.

8. Lámina con segmentos casi iguales, ninguno divido hasta la base........... pedatoradiatum subsp. pedatoradiatum 8. Lámina con segmentos desiguales, por lo menos el segmento intermedio libre hasta la base.

6. Láminas foliares simples y enteras.

A. pedatoradiatum subsp. helleborifolium

9. Láminas foliares cordadiformes o subcordiformes en la base.

10. Láminas conspicuamente punteadas de negro sobre el envés.

11. Láminas mayormente de 2 a 3 veces más largas que anchas, el lobo anterior usualmente más o menos oblongo y algunas veces estrechado en la base a estrechamente triangular; inflorescencia usualmente más larga que las hojas; espádice alargado (10-25 cm largo)

A. verapazense

11. Láminas mayormente 1.25 a 1.5 veces más largas que anchas, raramente más que dos veces más largas que anchas, el lobo anterior usualmente más o menos ovado, raramente con el lobo anterior un tanto oblongo o estrechamente triangular; inflorescencia usualmente más corta que las hojas; espádice relativamente corto (10.5$13 \mathrm{~cm}$ de largo).

A. lucens

10. Láminas sin puntuación negra sobre el envés.

12. Pecíolos afiladamente en forma de D; láminas más de $60 \mathrm{~cm}$ ancho.

A. faustomirandae

12. Pecíolos teretes a subteretes; láminas menos de $40 \mathrm{~cm}$ de ancho.

13. Pedúnculos menos de 1.3 veces tan largos como los pecíolos; espádice violeta rojizo a color lavanda; lóbulos basales de la lámina casi redondeados, dirigidos hacia la base. A. huixtlense 13. Pedúnculos más corto que los pecíolos; espádice verde; lóbulos basales de la lámina estrechos y dirigidos hacia afuera. A. berriozabalense

9. Láminas foliares no cordiformes o subcordiformes en la base, o al menos con los lobos posteriores menos que $1 / 4$ del largo de los lobos anteriores.

14. Plantas con entrenudos muy cortos y con las raíces densas, casi contiguas; hojas dispuestas en una roseta; láminas obovadas-elípticas, generalmente más anchas por arriba de la mitad.

A. schlechtendalii subsp. schlechtendali

14. Plantas con entrenudos alargados y con las raíces ampliamente dispersas sobre el tallo; hojas nunca dispuestas en una roseta; láminas elípticas a estrechamente elípticas, más anchas cerca del medio.

15. Láminas punteado-glandulares oscuras sobre el envés; bayas violeta pálido a casi blancas; semillas usualmente 4 o más por baya, amarillo pálidas, ca. $2 \mathrm{~mm}$ de largo. A. scandens subsp. scandens

15. Láminas no punteadas sobre el envés; bayas anaranjadas a rojas; semillas usualmente 2 por baya, negras, 6-9 mm de largo...... A. flexile subsp. flexile

5. Pecíolos envainados casi hasta la mitad o completamente. 
Apéndice 1. Continuación.

16. Láminas siempre enteras; pecíolos usualmente casi o completamente envainados, vaina persistente a marcescente; semillas redondas-reniformes, aplanadas, menos de $1.6 \mathrm{~mm}$ de largo, endosperma presente...... Rhodospatha wendlandii

16. Láminas a menudo perforadas, raramente profundamente pinatífidas o enteras; pecíolos envainados, vaina no siempre persistente, descomponiéndose en fibras o masas membranosas o enteramente decidua; semillas subglobosas a oblongas, 6-22 mm largo, endosperma ausente. Monstera

17. Láminas adultas con nervios secundarios completamente reticulados, ovado-cordadas en el contorno, con lobos posteriores redondeados; plantas juveniles carentes de hojas imbricadas.

18. Plantas hemiepífitas no robustas, trepadoras, pendientes al comienzo de la floración; tallos delgados, epidermis amarillo-marrón la cual se defolia o descascara; láminas más largas que los pecíolos; inflorescencias pendientes.

M. tuberculata var. tuberculata 18. Plantas hemiepífitas robustas adpreso-trepadoras; no pendientes al comienzo de la floración; tallos gruesos, sin epidermis amarillo-marrón; láminas más cortas que los pecíolos; inflorescencias erectas.........

M. deliciosa

17. Láminas adultas con nervios laterales secundarios paralelos (por lo menos de la distancia desde el margen), estrechamente ovadas a oblongo-elípticas, no típicamente ovado-cordadas, comúnmente sin lobos posteriores; plantas juveniles con o sin hojas imbricadas.

19. Pedúnculos más cortos que el espádice; plantas juveniles con hojas imbricadas; láminas a menudo con perforaciones grandes y no tan concentradas en medio de la lámina o cerca del nervio medio.....

M. acuminata

19. Pedúnculos más largo que el espádice; plantas con hojas no imbricadas; láminas a menudo con perforaciones mayormente pequeñas y concentradas en medio de la lámina más cerca del nervio medio.

M. egregia

2. Espádice no uniforme, flores unisexuales (dividido en flores masculinas en el ápice y flores femeninas en la base, con una zona de flores estériles masculinas entre ellas), espata envolviendo al espádice, prominentemente convoluta en la base.

20. Lámina de la espata volviéndose a cerrar sobre la parte estaminada del espádice después de la antesis.

21. Parte pistilada del espádice fusionada a la espata; flores pistiladas muy separadas entre sí, cada una rodeada por estaminodios delgados

Dieffenbachia oerstedii

21. Parte pistilada del espádice libre de la espata excepto en o cerca de su base; flores pistiladas casi contiguas, estaminodios ausentes.

Philodendron

22. Plantas adultas con pecíolos envainados solo cerca de la base, la vaina no rodea al tallo; láminas de las hojas muy variables en forma (cordiformes o sagitadas)..........Philodendron subgen. Philodendron 23. Plantas terrestres; tallos generalmente rastreros sobre el suelo; inflorescencias glabras .P. glanduliferum var. glanduliferum

23. Plantas trepadoras-hemiepífitas.

24. Láminas con márgenes profundamente divididos, profundamente pinnado lobados o trilobados a trifoliados.

25. Láminas profundamente pinnado lobadas con las divisiones extendiéndose más de 7/10 hacia el nervio medio. $P$. radiatum var. radiatum

25. Láminas profundamente trilobadas o trifoliadas. P. tripartitum

24. Láminas enteras con márgenes no profundamente divididos o al menos con los márgenes simplemente sinuados, los márgenes laterales del lobo anterior a veces profundamente cóncavos pero la lámina nunca trilobada.

26. Plantas hemiepífitas con entrenudos usualmente mucho más largos que anchos, tallos colgantes (excepto en P. smithii).

27. Tallos toscamente ásperos, conspicuamente setoso-pubescentes; láminas delgadas

27. Tallos glabros, láminas subcoriáceas a coriáceas.

P. jacquinii

28. Láminas estrechamente ovado-sagitadas; seno típicamente hipocrateriforme, a veces espatulado; pecíolos esponjosos; tallos por lo general grisáceos a café claro lustrosos .P. smithii 
Apéndice 1. Continuación.

28. Láminas ovadas; seno típicamente espatulado, raramente hipocrateriforme; pecíolos no esponjosos; tallos verde oscuros.

29. Láminas juveniles aterciopeladas, a menudo teñida de púrpura en el envés

P. hederaceum var. hederaceum

29. Láminas juveniles no aterciopeladas, nunca teñidas de púrpura en el envés, por lo general solo brillantes en el haz y verdes en el envés .P. hederaceum var. oxycardium

26. Plantas hemiepífitas con entrenudos usualmente más anchos que largos, tallos a menudo no colgantes.

30. Láminas estrechamente ovadas; catáfilos persistentes en restos fibrosos; 2-6 inflorescencia por axila. $P$. jodavisianum

30. Láminas ovada-cordadas, sagitadas a ovada-triangular; catáfilos deciduos; menos de 5 inflorescencias por axila.

31. Inflorescencia con espata no marcadamente constreñida cerca de la mitad o arriba de la porción del tubo; porción estaminada estéril del espádice frecuentemente (50\%) más ancha que la porción pistilada en antesis. P. advena

31. Inflorescencia con espata visiblemente constreñida arriba del tubo (usualmente un poco por debajo del medio); porción estaminada estéril del espádice más angosta hasta ligeramente más ancha que la porción pistilada en antesis. P. sagittifolium

22. Plantas adultas con pecíolos envainados en casi todo su largo y con vainas unidas circunferencialmente a los tallos; láminas de las hojas mayormente oblongas a elípticas.....

Philodendron subgen. Pteromischum

32. Plantas con raíces adventicias en la mayoría de los nudos; inflorescencias (1-) 2 (-3); catafilos típicamente presentes; pedúnculo usualmente más de 1/3 de la longitud del espádice

Philodendron subgen. Pteromischum Secc. Pteromischum

33. Pecíolo envainado en un $75 \%$ o menos de su longitud, la vaina estrictamente erecta; porción del pecíolo no envainada $7.4-29.3 \mathrm{~cm}$ de largo; plantas terrestres o rupícolas.

P. escuintlense

33. Pecíolo envainado $75 \%$ o más de su longitud, la vaina frecuentemente se vuelve horizontal extendida distalmente; porción del pecíolo no envainada (0.9) 2.0-8.1 cm de largo; plantas hemiepífitas .P. standleyi 32. Plantas sin raíces adventicias en la mayoría de los nudos; inflorescencias generalmente solitarias (excepto en $P$. inequilaterum); catafilos raramente presentes; pedúnculo comúnmente menos de $1 / 3$ de la longitud del espádice.

Philodendron subgen. Pteromischum Secc. Fruticosa

34. Vaina del pecíolo generalmente involuta a erecto-patente; inflorescencias frecuentemente apareadas; semillas frescas blancas........................... inequilaterum subsp. inequilaterum 34. Vaina del pecíolo involuta a horizontalmente entendida; inflorescencias mayormente solitarias; semillas típicamente rojas o púrpuras.

35. Hojas a menudo cordadas en la base; superficie externa de la espata con conductos resiníferos marrones finamente paralelos cuando seca.........P. schottii subsp. talamancae 35. Hojas a menudo cuneadas a cordadas en la base; superficie externa de la espata no conspicuamente resinoso-estriada.

36. Tallos secando de marrón-café a negruzcos; envés de la hoja con largos canales resiníferos conspicuos a lo largo de los nervios laterales; pedúnculo 1.0-2.8 cm de largo

..P. tuerkheimii

36. Tallos secando de amarillo claro a marrón oscuro; envés de la hoja sin canales resiníferos conspicuos, pedúnculo 1.3-3.2 cm de largo, 0.13-0.26 del largo de la espata.....

P. seguine subsp. lingua-bovis

20. Lámina de la espata no volviéndose a cerrar sobre la parte estaminada del espádice después de la antesis, por el contrario cayendo libremente o desprendiéndose. 
Apéndice 1. Continuación.

37. Plantas terrestres, robustas con láminas de $c a$. 1 m o más de largo; láminas triangularsagitadas; inflorescencias hasta 6 por axilas. Xanthosoma robustum 37. Plantas hemiepífitas; láminas de menos de $1 \mathrm{~m}$ de largo; láminas juveniles simple y hastadas; láminas adultas pedatamente lobadas; inflorescencias 1 o varias por axilas Syngonium

38. Hojas adultas enteras, ovado-cordadas o trilobadas, con los lobos laterales confluentes con el lobo medio ampliamente ovado. S. chiapense 38. Hojas adultas palmaticompuestas, 5-11-lobadas, los segmentos laterales conspicuamente auriculados.

39. Inflorescencias generalmente una (a veces 2 o 3); tubo de la espata no glauco; parte estaminada del espádice $8.5-14 \mathrm{~cm}$ largo. S. neglectum 39. Inflorescencias generalmente 4-11; tubo de la espata a veces glauco; parte estaminada del espádice $2.5-11.1 \mathrm{~cm}$ largo.

40. Lámina adulta coriácea; haz muy liso; segmento medio frecuentemente más de $35 \mathrm{~cm}$ de largo; láminas juveniles cordiformes; pedúnculos de 10-20 cm de largo ..S. macrophyllum

40. Lámina adulta delgada; haz no liso; segmento medio menos de $35 \mathrm{~cm}$ de largo; láminas juveniles delgadas, sagitadas o hastadas; pedúnculos menos de $10 \mathrm{~cm}$ de largo.

41. Tallos no glaucos, a veces con numerosas emergencias ásperas; segmentos de las hojas generalmente estrechos, o casi igual en tamaño y forma; flores estaminadas profundamente retusas en el ápice, sin indicación de la línea de fusión, espata persistente después de la antesis S. angustatum 41. Tallos glaucos, lisos, sin emergencias; segmentos de las hojas generalmente desiguales en tamaño y forma; flores estaminadas truncadas en el ápice y con indicación de la línea de fusión, espata caduca después de la antesis

..S. podophyllum var. podophyllum 
Diversidad florística de Araceae en el estado de Tabasco, México

Apéndice 2. Listado de Araceae presentes en el estado de Tabasco, indicando forma de vida (acu: acuática flotante, ep: holoepífita, hep: hemiepífita, rup: rupícola, ter: terrestre) y tipo de vegetación donde se encuentran ( $\mathrm{CH}$ : comunidades de hidrófitas, ET: encinar tropical, MGR: manglar, PZL: pastizal, SAP: selva alta perennifolia, SMP: selva mediana perennifolia, SMS: selva mediana subperennifolia, VR: vegetación riparia, VS: vegetación secundaria. Intervalo altitudinal de las Araceae presentes en Tabasco, intervalo altitudinal y distribución en el Neotrópico (ANT: Antillas, BLZ: Belice, BOL: Bolivia, BRA: Brasil, COL: Colombia, CRI: Costa Rica, CUB: Cuba, ECU: Ecuador, SVD: El Salvador, GTM: Guatemala, GUY: Guyana, GNF: Guayana francesa, HON: Honduras, ISC: Islas Caimán, MEX: México, NRG: Nicaragua, PAN: Panamá, SRM: Surinam, TYT: Trinidad y Tobago, VEN: Venezuela). ${ }^{+}$Nuevo registro para el estado de Tabasco; * Especie endémica de México; Tipo de vegetación de acuerdo a la clasificación de López (1980); 1,2 Datos obtenidos de Acebey y Krömer (2008) y Tropicos (2012).

\begin{tabular}{|c|c|c|c|c|c|}
\hline Especie & $\begin{array}{l}\text { Forma } \\
\text { de vida }\end{array}$ & $\begin{array}{c}\text { Tipo de } \\
\text { Vegetación }\end{array}$ & $\begin{array}{c}\text { Intervalo } \\
\text { altitudinal } \\
\text { en Tabasco } \\
\text { (msnm) }\end{array}$ & $\begin{array}{c}\text { Intervalo } \\
\text { altitudinal en } \\
\text { el Neotrópico } \\
(\mathbf{m s n m})^{1,2}\end{array}$ & $\begin{array}{l}\text { Distribución } \\
\text { en el } \\
\text { Neotrópico }^{1,2}\end{array}$ \\
\hline Anthurium berriozabalense Matuda ${ }^{+}$ & rup, ter & SAP, SMS & $60-80$ & $\begin{array}{c}1,000-1,300 \\
(-1,500)\end{array}$ & MEX, BLZ, GTM \\
\hline $\begin{array}{l}\text { Anthurium faustomirandae Pérez-Farrera } \\
\text { \& } \text { Croat }^{*+}\end{array}$ & rup & SAP & 575 & $550-860$ & MEX \\
\hline Anthurium flexile Schott subsp. flexile & hep & SAP, SMP, SMS, VR & $0-700$ & $0-1,000$ & MEX-PAN \\
\hline Anthurium huixtlense Matuda ${ }^{+}$ & ep & SAP & $700-1,000$ & $0-2,849$ & MEX-NRG \\
\hline Anthurium lucens Standl. ex Yuncker ${ }^{+}$ & ep & SAP, SMP, SMS & $100-700$ & $100-2,000$ & MEX-PAN \\
\hline $\begin{array}{c}\text { Anthurium pedatoradiatum subsp. } \\
\text { helleborifolium (Schott) Croat* }\end{array}$ & ter & SAP, SMP, SMS & $20-720$ & $20-550$ & MEX \\
\hline $\begin{array}{l}\text { Anthurium pedatoradiatum Schott } \\
\text { subsp. pedatoradiatum }\end{array}$ & ter & SAP, VR, VS & $100-400$ & $0-800$ & MEX, GTM \\
\hline $\begin{array}{l}\text { Anthurium pentaphyllum var. } \\
\text { bombacifolium (Schott) Madison }\end{array}$ & hep & $\begin{array}{l}\text { ET, SAP, SMP, } \\
\text { SMS, VS }\end{array}$ & $0-700$ & $0-800$ & MEX-PAN \\
\hline $\begin{array}{l}\text { Anthurium scandens (Aubl.) Engl. } \\
\text { subsp. scandens }\end{array}$ & ep & $\begin{array}{l}\text { PZL, SAP, SMS, } \\
\quad V R, V S\end{array}$ & $50-1,000$ & $0-2,700$ & $\begin{array}{l}\text { MEX-BOL, } \\
\text { BRA, ANT }\end{array}$ \\
\hline $\begin{array}{l}\text { Anthurium schlechtendalii Kunth } \\
\text { subsp. schlechtendalii }\end{array}$ & ep & $\begin{array}{l}\text { ET, MGR, PZL, SAP, } \\
\text { SMP, SMS, VR, VS }\end{array}$ & $0-300$ & $0-1,000(-1,600)$ & MEX-HON \\
\hline Anthurium verapazense Engl. & ep & SMP & 250 & $100-800(-1,400)$ & MEX, BLZ, GTM \\
\hline Dieffenbachia oerstedii Schott & ter & SAP, SMP, SMS, VR & $0-200$ & $0-1,260$ & MEX-PAN \\
\hline Monstera acuminata K. Koch & hep & $\begin{array}{l}\text { PZL, SAP, SMS, } \\
\quad \text { VR, VS }\end{array}$ & $0-800$ & $0-800$ & MEX-HON \\
\hline Monstera deliciosa Liebm. & hep & SAP, VS & $500-600$ & $400-2,000$ & MEX-PAN \\
\hline Monstera egregia Schott*+ & hep & SAP, SMS & $100-1,000$ & $100-1,500$ & MEX \\
\hline Monstera sp. 1 & hep & SAP & 720 & & \\
\hline $\begin{array}{l}\text { Monstera tuberculata Lundell var. } \\
\text { tuberculata }\end{array}$ & hep & $\begin{array}{l}\text { SAP, SMP, SMS, } \\
\text { VR, VS }\end{array}$ & $0-600$ & $0-500$ & MEX, BLZ, GTM \\
\hline Philodendron advena Schott & hep & SAP, SMS & $70-300$ & $70-2,500$ & $\begin{array}{l}\text { MEX, BLZ, GTM, } \\
\text { CRI, COL }\end{array}$ \\
\hline Philodendron escuintlense Matuda*+ & rup, ter & SAP & 575 & $150-500$ & MEX \\
\hline $\begin{array}{l}\text { Philodendron glanduliferum Matuda } \\
\text { subsp. glanduliferum }\end{array}$ & ter & SAP & $700-1,000$ & $600-1,900$ & MEX, GTM \\
\hline $\begin{array}{l}\text { Philodendron hederaceum (Jacq.) Schott } \\
\text { var. hederaceum }\end{array}$ & hep & SAP, SMS, VR, VS & $0-600$ & $0-1,200(-1,500)$ & $\begin{array}{c}\text { MEX-BOL, ANT, } \\
\text { BRA, VEN, } \\
\text { Guayanas }\end{array}$ \\
\hline $\begin{array}{l}\text { Philodendron hederaceum var. } \\
\text { oxycardium (Schott) Croat* }\end{array}$ & hep & SAP, SMS & $0-300$ & $100-600$ & MEX \\
\hline $\begin{array}{l}\text { Philodendron inaequilaterum Liebm. } \\
\text { subsp. inaequilaterum }\end{array}$ & hep & SAP, SMP, SMS & $0-300$ & $0-1,500(-1,820)$ & $\begin{array}{l}\text { MEX-COL, VEN, } \\
\text { ECU }\end{array}$ \\
\hline
\end{tabular}


Pedro DíaZ-Jiménez ET AL.

Apéndice 2. Continuación.

\begin{tabular}{|c|c|c|c|c|c|}
\hline Especie & $\begin{array}{l}\text { Forma } \\
\text { de vida }\end{array}$ & $\begin{array}{c}\text { Tipo de } \\
\text { Vegetación }\end{array}$ & $\begin{array}{c}\text { Intervalo } \\
\text { altitudinal } \\
\text { en Tabasco } \\
(\text { msnm) }\end{array}$ & $\begin{array}{c}\text { Intervalo } \\
\text { altitudinal en } \\
\text { el Neotrópico } \\
(\mathrm{msnm})^{1,2}\end{array}$ & $\begin{array}{l}\text { Distribución } \\
\text { en el } \\
\text { Neotrópico }^{1,2}\end{array}$ \\
\hline Philodendron jacquinii Schott & hep & ET, SMS, VR, VS & $0-300$ & $0-1,500(-2,500)$ & $\begin{array}{c}\text { MEX-PAN, COL, } \\
\text { VEN, Guayanas, } \\
\text { CUB, ISC }\end{array}$ \\
\hline Philodendron jodavisianum G.S. Bunting & hep & SMS & $50-300$ & $0-1,500$ & $\begin{array}{l}\text { MEX-COL, } \\
\text { VEN, ECU }\end{array}$ \\
\hline $\begin{array}{l}\text { Philodendron radiatum Schott } \\
\text { var. radiatum }\end{array}$ & hep & SAP, SMS, VR, VS & $0-600$ & $0-700$ & MEX-COL \\
\hline Philodendron sagittifolium Liebm. & hep & SAP, SMS & $0-800$ & $0-1,800$ & MEX-COL \\
\hline $\begin{array}{l}\text { Philodendron schottii subsp. talamancae } \\
\text { (Engl.) Grayum }\end{array}$ & hep & SAP, SMP, SMS & $60-70$ & $0-1,200(-1,500)$ & MEX-ECU \\
\hline $\begin{array}{l}\text { Philodendron seguine subsp. lingua-bovis } \\
\text { Grayum*+ }\end{array}$ & hep & SAP & 750 & $0-1,100(-1,400)$ & MEX \\
\hline Philodendron smithii Engl. & hep & SAP, SMP, SMS & $0-300$ & $40-1,630$ & $\begin{array}{l}\text { MEX, BLZ, GTM, } \\
\text { HON, NRG }\end{array}$ \\
\hline Philodendron sp. 1 & hep & SAP & 750 & & \\
\hline Philodendron standleyi Grayum ${ }^{+}$ & hep & SAP & 1,028 & $\begin{array}{c}(400-) 600-1,800 \\
(-2,100)\end{array}$ & MEX-PAN \\
\hline Philodendron tripartitum (Jacq.) Schott & hep & SAP, SMP, SMS, VS & $0-800$ & $0-1,300(-1,500)$ & MEX-ECU \\
\hline Philodendron tuerckheimii Grayum & hep & SAP, SMS & $50-800$ & $0-2,050$ & MEX-ECU, VEN \\
\hline Pistia stratiotes L. & $\mathrm{acu}$ & $\mathrm{CH}$ & $0-100$ & $0-1,260$ & Pantropical \\
\hline $\begin{array}{l}\text { Rhodospatha wendlandii Schott var. } \\
\text { wendlandii }\end{array}$ & hep & SAP, SMS & $0-800$ & $0-550(-1,600)$ & MEX-COL, VEN \\
\hline Spathiphyllum blandum Schott & ter & SMS & 60 & $0-1,900$ & $\begin{array}{l}\text { MEX, BLZ, GTM, } \\
\text { HON }\end{array}$ \\
\hline $\begin{array}{l}\text { Spathiphyllum cochlearispathum } \\
\text { (Liebm.) Engl. }\end{array}$ & ter & $\begin{array}{l}\text { SAP, SMP, SMS, } \\
\text { VR, VS }\end{array}$ & $0-1,000$ & $0-1,600$ & $\begin{array}{l}\text { MEX, BLZ, GTM, } \\
\text { HON, CRI }\end{array}$ \\
\hline Syngonium angustatum Schott & hep & $\begin{array}{l}\text { ET, MGR, PZL, SAB, } \\
\text { SMP, SMS, VR, VS }\end{array}$ & $0-300$ & $0-700(-1,200)$ & MEX-CRI \\
\hline Syngonium chiapense Matuda & hep & SAP, VS & $100-800$ & $0-1,200(-1,500)$ & MEX, GTM \\
\hline Syngonium macrophyllum Engl. & hep & SAP, SMP, SMS & $0-700$ & $0-1,100$ & MEX-ECU \\
\hline Syngonium neglectum Schott* & hep & SAP & 50 & $(0-) 350-1,700$ & MEX \\
\hline $\begin{array}{l}\text { Syngonium podophyllum Schott var. } \\
\text { podophyllum }\end{array}$ & hep & $\begin{array}{c}\text { PZL, SAB, SAP, SMP, } \\
\text { SMS, VR, VS }\end{array}$ & $0-200$ & $0-1,000(-1,500)$ & $\begin{array}{l}\text { MEX-BOL, BRA, } \\
\text { GNF }\end{array}$ \\
\hline Xanthosoma robustum Schott & ter & SAP, SMS, VS & $0-600$ & $0-1,400$ & MEX-CRI \\
\hline
\end{tabular}

